\title{
NUEVAS FUENTES DE ANTIOXIDANTES NATURALES: CARACTERIZACIÓN DE COMPUESTOS BIOACTIVOS EN CINCO FRUTOS NATIVOS DE CHILE.
}

\section{New source of natural antioxidants: Characterisation of bioactive compounds in five native Chilean fruits}

${ }^{1,2}$ ME. Romero Román*, ${ }^{1}$ F. Noriega Vásquez, ${ }^{1}$ M. Farías Villagra, ${ }^{1} \mathrm{P}$. Jara Zapata, ${ }^{1}$ B. Vera Flores, ${ }^{1}$ MD. López Belchi

${ }^{1}$ Universidad de Concepción, Facultad de Agronomía, Departamento de Producción Vegetal, Laboratorio de Análisis Químico, Concepción, Chile

${ }^{2}$ Universidad Técnica de Babahoyo, Facultad de Agronomía, Departamento de Biotecnología, Babahoyo, Ecuador

*mariaeugeromero@udec.cl

Diferentes berries de la zona centro y sur de Chile fueron analizados con el fin de buscar fuentes promisorias de polifenoles con clara actividad sobre la salud humana. Se estudiaron cinco bayas nativas: arrayan, frutilla blanca, murtilla y calafate, y un berry tradicional (uva tintorera). Se determinó in vitro sus propiedades antioxidantes según el ensayo de polifenoles totales de Folin Ciocalteu, antocianinas por $\mathrm{pH}$ diferencial, capacidad antioxidante por medición de capacidad de reducción del radical libre 2.2-difenil-1picrilhidracilo (DPPH) y poder de reducción férrica (FRAP) y perfil químico por HPLC-DAD. De los cinco berries, calafate registró el valor más alto (1066,4 $\pm 24,9 \mathrm{mg} \mathrm{GAE} / 100 \mathrm{~g}$ de muestra) para polifenoles totales y antocianinas (1031,9 \pm 48,1 mg de cianidina-3-glucósido/100g de muestra) seguido por uva tintorera. Calafate presentó excelente poder reductor $(11279,2 \pm 2027,4 \mu \mathrm{mol}$ Trolox $/ 100 \mathrm{~g}$ ensayo FRAP y 5235,0 \pm $445,9, \mu \mathrm{mol} / 100 \mathrm{~g}$ en $\mathrm{DPPH})$. El perfil químico de antocianinas mostró delfinidina, cianidina, malvidina, petunidina, peonidina y pelargonidina en los cinco berries. Alrededor de 30 flavonoles derivados de quercetina, myricetina e isorhamnetina fueron identificados así cómo elagitaninos presentes en frutilla blanca, compuestos muy interesantes para estudios posteriores. Estos resultados contribuyen a destacar el uso potencial de estos berries como alimentos funcionales.

Palabras clave: berries, polifenoles, antocianinas, capacidad antioxidante, HPLC-DAD.

Berries from central and southern Chile were analyzed in order to find promising sources of polyphenols with clear activity on human health. Five native fruits like arrayan, white strawberry, murtilla and calafate, and a traditional berry (grape called "tintorera") were studied. Antioxidant properties were determined in vitro according to the total polyphenol assay of Folin Ciocalteu, anthocyanins by differential $\mathrm{pH}$, antioxidant capacity by measuring the capacity of reduction of the free radical 2.2-diphenyl-1-picrylhydracil (DPPH) and iron reducing capacity (FRAP) and chemical profile by HPLC-DAD. Calafate showed the highest value $(1066.4 \pm 24.9 \mathrm{mg}$ gallic acid / $100 \mathrm{~g}$ sample) for total polyphenols and anthocyanins (1031.9 $\pm 48.1 \mathrm{mg}$ of cyanidin-3-glucoside / $100 \mathrm{~g}$ of sample) followed by blue grape. Calafate displayed excellent reducing power (11279.2 \pm 2027.4 $\mu \mathrm{mol}$ Trolox / 100g FRAP assay and 5235.0 $\pm 445.9, \mu \mathrm{mol} / 100 \mathrm{~g}$ in DPPH), followed by grape. The chemical profile of anthocyanins showed delphinidin, cyanidin, malvidin, petunidin, peonidin and pelargonidin in the five berries. Around 30 flavonols derived from quercetin, myricetin and isorhamnetin were identified as well as ellagitannins present in white strawberry, very interesting compounds for further studies. These results contribute to highlight the potential use of these berries as functional foods.

Key words: berries, polyphenols, anthocyanins, antioxidant capacity, HPLC-DAD. 


\section{INTRODUCCIÓN}

La relación entre salud y nutrición ha puesto su atención en compuestos bioactivos de los alimentos que puedan mejorar las condiciones y calidad de vida (1). La principal fuente de nutrientes son las frutas, las cuales aportan vitaminas, minerales, proteína y fibra. Por otro lado, gracias al metabolismo secundario, las plantas producen compuestos como carotenoides, esteroles, glucosinolatos, polifenoles, entre otros; $(2,3)$ que le sirven para protegerse frente a factores bióticos y abióticos (4) y para la comunicación alelopática (5). Las bayas o "berries" son frutas caracterizadas por ser aromáticas y poseer vistosos colores. Estas características son otorgadas por algunos de los metabolitos secundarios antes mencionados, producidos por estas frutas como mecanismo de protección frente a radiación y baja temperatura $(5,6)$. En diversos estudios se ha determinado que dichos compuestos brindan beneficios claros en nuestra salud, ya que tienen la capacidad de evitar el estrés oxidativo (7).

Si se considera el contenido de compuestos bioactivos, a la presente fecha, la promoción del consumo de frutos rojos es creciente, ya que los compuestos químicos que ellas producen, están asociados con la protección frente a enfermedades degenerativas (8), crónicas (9), inflamatorias (10), entre otras. En Chile, existe una amplia diversidad de bayas que crecen bajo distintas condiciones ecológicas (11), sin embargo, algunas como el arrayán o arrayana (Luma apiculata) (12), calafate (Berberis microphylla) (13), frutilla blanca (Fragaria chiloensis ssp. chiloensis) (14) y murtilla (Ugni molinae) (15) que, por su condición silvestre o de semi-domesticación, su valor a nivel agronómico, industrial, y nutracéutico aún no ha sido explotado. A pesar de no ser nativa, otra baya de importancia por su representatividad en la cultura vitivi- nícola chilena es la uva tintorera, que es uno de los pocos cultivares de Vitis vinifera con una pulpa de bayas de color rojo, (cultivar teinturier) y que por su alto potencial de intensidad de color se usa para mejorar vinos menos coloreados (16).

En la mayoría de los berries comerciales se han encontrado antocianinas como delfinidina glicosilada, petunidina, malvidina, peonidina y cianidina (17-19) , así como ácido elágico, y elagitaninos en frutillas y fresas (20); muchos de ellos están asociados con los efectos de los beneficios de salud (9). La absorción de fuentes de polifenoles asegura la eliminación de radicales libres y también aumenta las propiedades cognitivas y previene o reduce el riesgo de enfermedades neurodegenerativas (21)

Por los antecedentes se conoce que las bayas tienen una producción considerable de metabolitos secundarios la misma que está condicionada por los factores edafoclimáticos que rodeen a la planta, así, el presente trabajo busca destacar el potencial de cinco berries nativos chilenos del centro y sur de Chile, para promoción de futuros trabajos y generación de alimentos funcionales en relación a los compuestos bioactivos de arrayan, calafate, murtilla, frutilla blanca y uva tintorera.

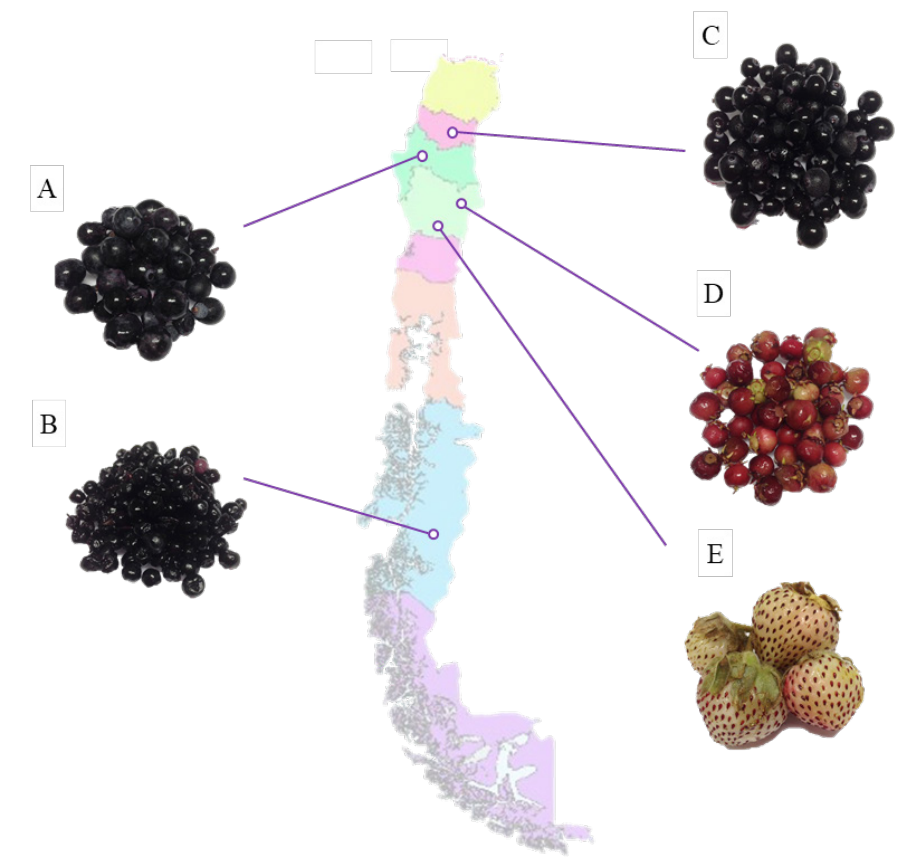

Figura 1. Berries en estudio y distribución en la zona centro y sur de Chile. A Uva tintorera (V. vinifera) B. Calafate (B. microphylla C. Arrayán (L. apiculata) D. Murtilla (U. molinae) E). Frutilla blanca (F. chiloensis ssp. chiloensis).

\section{MATERIALES Y MÉTODOS}

\section{Material vegetal}

Se trabajó con bayas en estado óptimo de maduración co- 
lectadas de la zona centro y sur de Chile: arrayana (Luma apiculata) de Mulchén, Región de Bio-Bio, calafate (Berberis microphylla) de Coyhaique, Región Aysén; frutilla blanca (Fragaria chiloensis ssp.chiloensis) de Purén, Región de la Araucanía, murtilla (Ugni molinae) de Temuco, Región de la Araucanía y tintorera (Vitis vinífera) de Quillón, Región de Nuble (Fig. 1); y se mantuvieron a $-80^{\circ} \mathrm{C}$ en el Laboratorio de Química, Departamento de Producción Vegetal de la Universidad de Concepción campus Chillan previo a su análisis.

\section{Reactivos}

Estándares comerciales de delfinidina-3-glucósido (98.84 \%), cianidina3-glucósido (99.21\%), petunidin-3-glucósido (99.90\%), malvidin-3-glucósido (99.91\%), Peonidin-3-glucósido (> $97 \%$ ), cianidina-3-lathyroside (> 97 \%) de Sigma -Aldrich. El reactivo Folin-Ciocalteu, carbonato sódico ( $\mathrm{Na} 2 \mathrm{CO} 3)$, buffer de cloruro de potasio pH 1 y buffer acetato de sodio $\mathrm{pH}$ 4,5, el radical 2,2-difenil-1-picrilhidracil (DPPH), Trolox, $\mathrm{FeCl} 3-6 \mathrm{H} 2 \mathrm{O}, \mathrm{HCl}$, TPTZ por Merck (Alemania) y ácido fórmico, acetonitrilo, metanol y agua en grado HPLC de Merk.

\section{Características físico-químicas y preparación de ex- tractos}

La determinación de las propiedades físico-químicas se realizó a partir de frutos elegidos al azar. Se midieron diámetros ecuatorial y polar de 20 berries por especie y el peso en 100 frutos de cada una de las muestras. El pH, solidos solubles y acidez se obtuvieron a partir de frutos triturados, utilizando el pHmetro digital marca INOLAB serie $\mathrm{PH} 7110$ para $\mathrm{pH}$ y la medición de solidos solubles en ${ }^{\circ}$ Brix utilizando un refractómetro SPER SCIENTIFIC serie 300010. La acidez se determinó por titulación con $\mathrm{NaOH} 0,1 \mathrm{M}$ a partir de $0,5 \mathrm{~g}$ de muestra triturada y diluida en $150 \mathrm{ml}$ de agua destilada. La extracción de antioxidantes se realizó por ultrasonido a partir de $0,5 \mathrm{~g}$ de muestra en $5 \mathrm{ml}$ de metanol/agua/acido fórmico (25:24:1 / v: v: v) con 1 hora de ultrasonido, incubación a $0^{\circ} \mathrm{C} \mathrm{du}-$ rante $24 \mathrm{~h}$ y posterior centrifugación a $3.500 \mathrm{rpm}$ durante 15 minutos.

\section{Determinación de polifenoles totales, antocianinas y capacidad antioxidante}

El análisis de polifenoles totales se realizó por el método de Folin Ciocalteu (22), con una curva de calibración de ácido gálico como estándar $(0,0.1,0,25 ; 0,5$ y 1 g/l) y medición de la absorbancia de las muestras a una longitud de onda de $760 \mathrm{~nm}$ a través de un espectrofotómetro UV/vis Thermo Scientific; agregando a cada vial $120 \mu \mathrm{l}$ de reactivo de Folin Ciocalteu 1N, $25 \mu \mathrm{l}$ del extracto (muestra), 1,62 $\mathrm{ml}$ de agua y finalmente $340 \mu \mathrm{l}$ de la disolución de carbonato de sodio al $20 \%$, y 2 horas de incubación en oscuridad. El contenido de polifenoles fue expresado en $\mathrm{mg}$. de equivalente ác. gálico $/ 100 \mathrm{~g}$ peso fresco de muestra.

Para la determinación de antocianinas totales se utilizó el ensayo de $\mathrm{pH}$ diferencial con tampones de cloruro de potasio $0,025 \mathrm{M}$ a pH 1 y acetato de sodio $0,4 \mathrm{M}$ a pH 4,5. (23) De cada extracto obtenido previamente se extrajeron dos muestras con $0,1 \mathrm{ml}$ en dos tubos por separado se agregó 2,9 ml de tampón $\mathrm{KCl}$ y al tubo 2 se adicionó 2,9 $\mathrm{ml}$ de tampón $\mathrm{CH} 3 \mathrm{CO}$ $2 \mathrm{Na}$ y se leyó la absorbancia a 510 y 700 nm de cada uno de los tubos (4 medidas espectrofotométricas en total) en un espectrofotómetro UV vis Thermo Scientific $^{\text {тx }}$. Los datos se expresaron en $\mathrm{mg}$ de cianidina-3-glucósido/100g de peso fresco.

El valor de capacidad antioxidante FRAP se obtuvo con $30 \mu \mathrm{l}$ del extracto, $300 \mu \mathrm{l}$ de agua destilada y la adición de $3000 \mu \mathrm{l}$ del reactivo FRAP ( $\mathrm{FeCl} 3-6 \mathrm{H} 2 \mathrm{O} 20 \mathrm{mM}$, TPTZ10mM y tampón acetato $0,3 \mathrm{mM}$ ) incubados a $37^{\circ} \mathrm{C}$ durante $30 \mathrm{~min}$ y medidos a $595 \mathrm{~nm}$ de absorbancia en el espectrofotómetro antes descrito. El blanco fue agua destilada. Los resultados se enunciaron como $\mu$ mol trolox $/ 100 \mathrm{~g}$ de muestra peso fresco. Respecto a la capacidad antioxidante DPPH, se utilizó 100 $\mu \mathrm{l}$ del extracto y $2,9 \mathrm{ml}$ de la solución DPPH agitados constantemente y posterior incubación en oscuridad durante 1 hora. Finalmente se realizó la lectura a $515 \mathrm{~nm}$. Los resultados se expresaron en $\mu \mathrm{mol}$ trolox en $100 \mathrm{~g}$ de muestra peso fresco. El blanco contenía $3 \mathrm{ml}$ de metanol.

Identificación y cuantificación de compuestos fenólicos por HPLC-DAD.

Los análisis HPLC-DAD para la identifi- 
cación y cuantificación de polifenoles se llevaron a cabo en una columna columna Kromolith de fase inversa (RP) -18 (250-4,6 mm id) equipada con un detector de fotodiodos (DAD) (Merk-Hitachi, Darmstadt, Alemania). con fase móvil compuesto por dos disolventes: agua (A) y ácido fórmico (B) $(99: 1, v / v)$ y caudal de $1 \mathrm{ml} /$ mincon variación del gradiente de $8 \%$ de disolvente $\mathrm{B}, 15 \%$ a $25 \mathrm{~min}$, $22 \%$ a $55 \min$ y $40 \%$ a $60 \mathrm{~min}$, mantenido hasta $70 \mathrm{~min}$. Los compuestos se identificaron por comparación con patrones. Para los análisis cuantitativos, se registraron los cromatogramas a 360 y 520 nm (Figura 2 y 3). Como estándares se utilizaron para las antocianinas cianidina-3-glucósido a $520 \mathrm{~nm}$ y para los flavonoles, quercetina-3-rutinósido a 360nm (Sigma Chemical Co. St. Louis, $\mathrm{MO})$.

Para determinar, polifenoles totales, antocianinas totales y capacidad antioxidante por los métodos FRAP y DPPH, cada muestra se replicó tres veces y los resultados se informan en medias \pm desviación estándar y análisis de varianza (ANOVA) con un nivel de significancia $\mathrm{p}<0,05$ y prueba de comparación de Tukey, utilizando el programa estadístico SAS versión 8.0 para Windows.

\section{RESULTADOS}

Las características físico-químicas y preparación de extractos se presentan en la Tabla 1 . Los resultados muestran que el $\mathrm{pH}$ de los berries está entre 3,7 y 4,2. siendo murtilla la más ácida y arrayan la más alcalina. Respecto a los azucares totales, la frutilla posee $7,5 \mathrm{~g} / 100 \mathrm{~g}$ por ende es la más dulce, seguido por calafate y uva tintorera. Estas características confirman la posibilidad de aceptación de estos berries para el consumo y aseguran el aprovechamiento de éstas en dietas sanas y/o la elaboración de alimentos funcionales.

En la Tabla 2, se registra el valor más alto $(1066,4 \pm 24,9$ mg ácido gálico /100g de muestra y 1031,9 $\pm 48,1 \mathrm{mg}$ de cianidina/100g de peso fresco) en polifenoles y antocianinas (respectivamente) en calafate superior a otros reportes (24). Los valores menores en cuanto a polifenoles, se obtuvieron para murtilla y frutilla blanca. Murtilla, presentan un total de 260,5 $\pm 20,2 \mathrm{mg}$ ác. gálico/100g muestra, similar a otro reporte (15), mientras que, frutilla blanca coincide con lo obtenido por otros investigadores (12).

Tabla 1 Comparación de características físico-químicas de arrayan (L. apiculata), uva tintorera ( $V$. vinifera), frutilla blanca (F. chiloensis ssp. chiloensis), murtilla (U. molinae) y calafate (B. microphylla) del centro y sur de Chile.

\begin{tabular}{|l|c|c|c|c|c|c|}
\hline \multicolumn{1}{|c|}{ Fruto } & Peso (mg.) & $\begin{array}{c}\text { Diámetro } \\
\text { Ecuatorial } \mathbf{( m m} \text {.) }\end{array}$ & $\begin{array}{c}\text { Diámetro } \\
\text { Polar }(\mathbf{m m} \text {.) }\end{array}$ & pH & ${ }^{\circ}$ Brix & $\begin{array}{c}\text { Azucares Totales } \\
\text { (g azúcar/100g) }\end{array}$ \\
\hline Calafate & $11,4 \pm 0,6 \mathrm{c}$ & $10,00 \pm 0,67 \mathrm{~cd}$ & $7,81 \pm 2,48 \mathrm{c}$ & $3,69 \pm 0,06 \mathrm{c}$ & $27,1 \pm 1,7 \mathrm{a}$ & $13,89 \pm 0,45 \mathrm{~b}$ \\
\hline Murtilla & $12,5 \pm 0,6 \mathrm{bc}$ & $9,93 \pm 0,61 \mathrm{~d}$ & $8,87 \pm 0,47 \mathrm{c}$ & $3,67 \pm 0,04 \mathrm{c}$ & $13,1 \pm 0,6 \mathrm{c}$ & $8,24 \pm 0,57 \mathrm{c}$ \\
\hline Uva & $14,0 \pm 0,6 \mathrm{bc}$ & $12,53 \pm 1,09 \mathrm{~b}$ & $14,02 \pm 0,95 \mathrm{~b}$ & $4,09 \pm 0,05 \mathrm{ab}$ & $23,6 \pm 1,0 \mathrm{~b}$ & $14,45 \pm 2,01 \mathrm{~b}$ \\
\hline Arrayan & $20,0 \pm 0,4 \mathrm{~b}$ & $12,12 \pm 1,68 \mathrm{bc}$ & $12,12 \pm 1,51 \mathrm{~b}$ & $4,21 \pm 0,08 \mathrm{a}$ & $9,6 \pm 0,4 \mathrm{~d}$ & $5,23 \pm 0,46 \mathrm{c}$ \\
\hline Frutilla blanca & $96,9 \pm 6,6 \mathrm{a}$ & $32,43 \pm 3,17 \mathrm{a}$ & $29,5 \pm 3,26 \mathrm{a}$ & $3,97 \pm 0,06 \mathrm{~b}$ & $11,6 \pm 0,4 \mathrm{~cd}$ & $40,74 \pm 2,12 \mathrm{a}$ \\
\hline
\end{tabular}

Tabla 1. ${ }^{*}$ Peso medido en $\mathrm{mg}$. y los diámetros polar y ecuatorial en $\mathrm{mm}$. Letras diferentes indican diferencias significativas entre valores de la misma columna $(\mathrm{p}>0.05)$.

En cuanto a capacidad antioxidante, los resultados (Tabla 2) demuestran que calafate posee el más alto poder reductor de radicales libre mediante método FRAP $(11279,2 \pm 2027,4 \mu \mathrm{mol} / 100 \mathrm{~g})$; similares datos han sido alcanzados previamente (13).

Para el radical DPPH, los mejores resultados son calafate $(5235,0 \pm 445,9$ a $\mu \mathrm{mol} / 100 \mathrm{~g}$.), arrayan $(4401,7 \pm 493,9)$ y uva tintorera $(4335,0 \pm 156,1 \mu \mathrm{mol} / 100 \mathrm{~g})$. Los valores para tintorera son superiores a lo reportado para uvas comerciales (27).

Tabla 2. Polifenoles totales, antocianinas totales, y capacidad antioxidante por medición del poder de reducción del radical libre DPPH y reducción férrica (FRAP) en arrayan (L. apiculata), uva tintorera (V. vinífera), frutilla blanca (F. chiloensis ssp. chiloensis), murtilla (U. molinae) y calafate (B. microphylla) del centro y sur de Chile. 


\begin{tabular}{|c|c|c|c|c|}
\hline Fruto & $\begin{array}{c}\text { Polifenoles Totales } \\
\text { mg ác. gálico/100g } \\
\text { muestra }\end{array}$ & $\begin{array}{c}\text { Antocianinas Totales } \\
\text { mg de cianidina/100g peso } \\
\text { fresco }\end{array}$ & $\begin{array}{c}\text { DPPH } \\
\mu \text { molTrolox } / 100 g \text { peso } \\
\text { fresco }\end{array}$ & $\begin{array}{c}\text { FRAP } \\
\mu \text { molTrolox/100g peso } \\
\text { fresco }\end{array}$ \\
\hline Calafate & $1066,4 \pm 24,9 \mathrm{a}$ & $1031,9 \pm 48,1 \mathrm{a}$ & $5235,0 \pm 445,9 \mathrm{a}$ & $11279,2 \pm 2027,4 \mathrm{a}$ \\
\hline Murtilla & $260,5 \pm 20,2 \mathrm{c}$ & $8,8 \pm 1,2 \mathrm{~d}$ & $2005,8 \pm 848,6 \mathrm{~b}$ & $5445,8 \pm 316,6 \mathrm{a}$ \\
\hline Uva tintorera & $639,5 \pm 41,8 \mathrm{~b}$ & $438,2 \pm 12,0 \mathrm{~b}$ & $4335,0 \pm 156,1 \mathrm{a}$ & $3262,5 \pm 288,3 \mathrm{bc}$ \\
\hline Arrayan & $464,4 \pm 31,6 \mathrm{c}$ & $287,7 \pm 11,4 \mathrm{c}$ & $4401,7 \pm 493,9 \mathrm{a}$ & $2104,2 \pm 344,9 \mathrm{c}$ \\
\hline Frutilla blan- & $273,4 \pm 18,4 \mathrm{c}$ & $3,8 \pm 0,4 \mathrm{~d}$ & $2800,8 \pm 175,3 \mathrm{~b}$ & $1343,0 \pm 250,0 \mathrm{c}$ \\
\hline ca & & & & \\
\hline
\end{tabular}

Tabla 2. ${ }^{\star}$ Letras diferentes indican diferencias significativas entre valores de la misma columna ( $\left.\mathrm{p}>0.05\right)$.
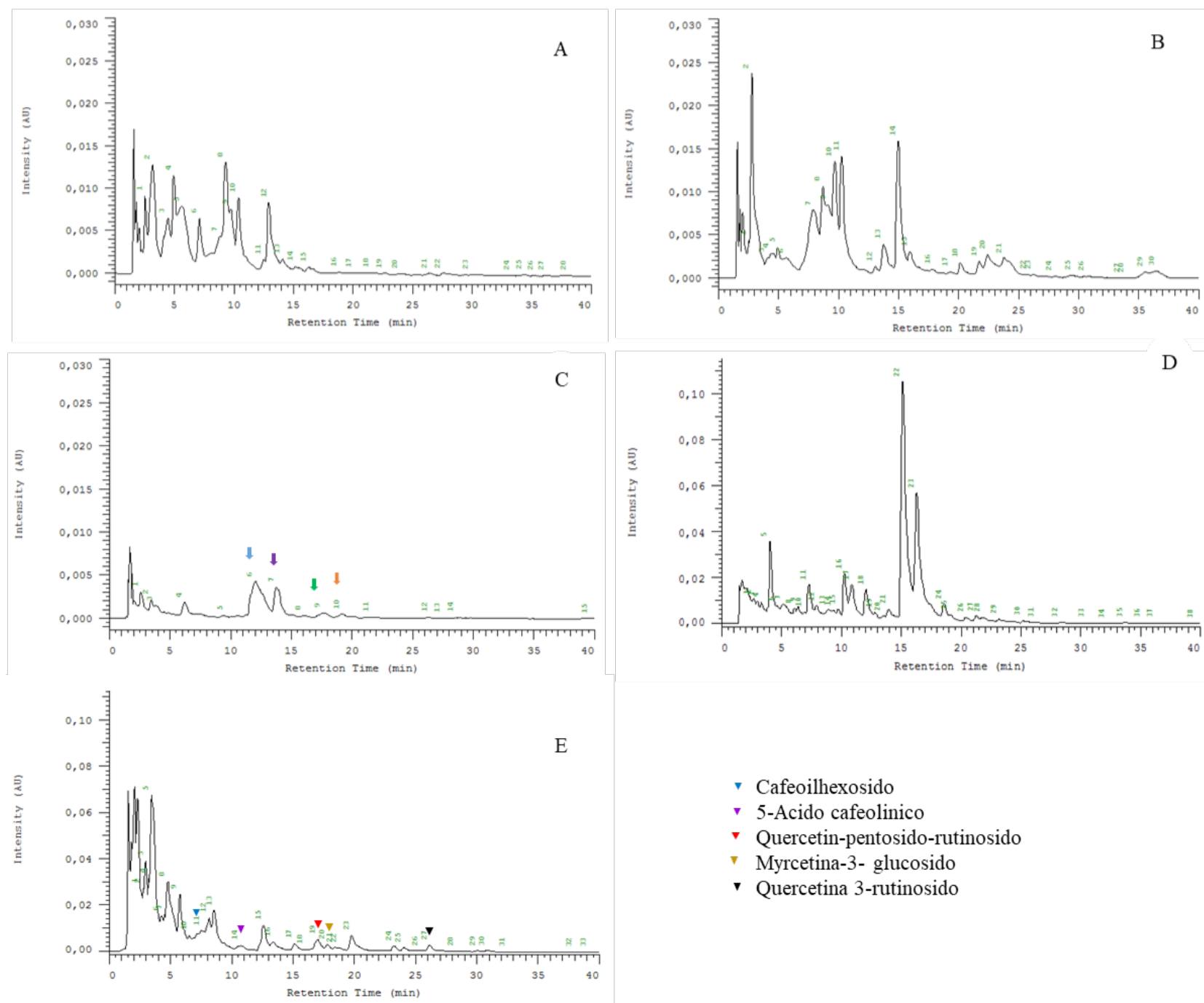

- Cafeoilhexosido

5-Acido cafeolinico

Quercetin-pentosido-rutinosido

Vyrcetina-3- glucosido

v Quercetina 3-rutinosido

Tabla 1. ${ }^{*}$ Peso medido en $\mathrm{mg}$. y los diámetros polar y ecuatorial en $\mathrm{mm}$. Letras diferentes indican diferencias significativas entre valores de la misma columna $(\mathrm{p}>0.05)$.

El perfil químico (Fig. 2), a longitud de onda 360nm muestra diferencias en los cromatogramas generando más de 30 posibles flavonoles. Se reportaron 26 flavonoles principalmente quercetina y myricetina para berries similares a los utilizados en este estudio (26). La Fig. 2-C muestra el cromatograma de frutilla blanca donde se han marcado compuestos que esta fruta posee pero que no se identificaron. Estos pudieran corresponder a elagitaninos (ácido elágico, glucósidos de ác. elágico y elagitaninos), además de pelargonidin-3-glucósido y cianidina-3-glucósido como describen otros investigadores (20) y similar a otro reporte donde a partir de fruta liofilizada se encontraron $2,12 \mathrm{mg}$ de quercetina/g. (27) como parte de los ácidos hidroxicinámicos (elagitaninos) y que presentan evitan daños cardiovasculares y cáncer (28). 


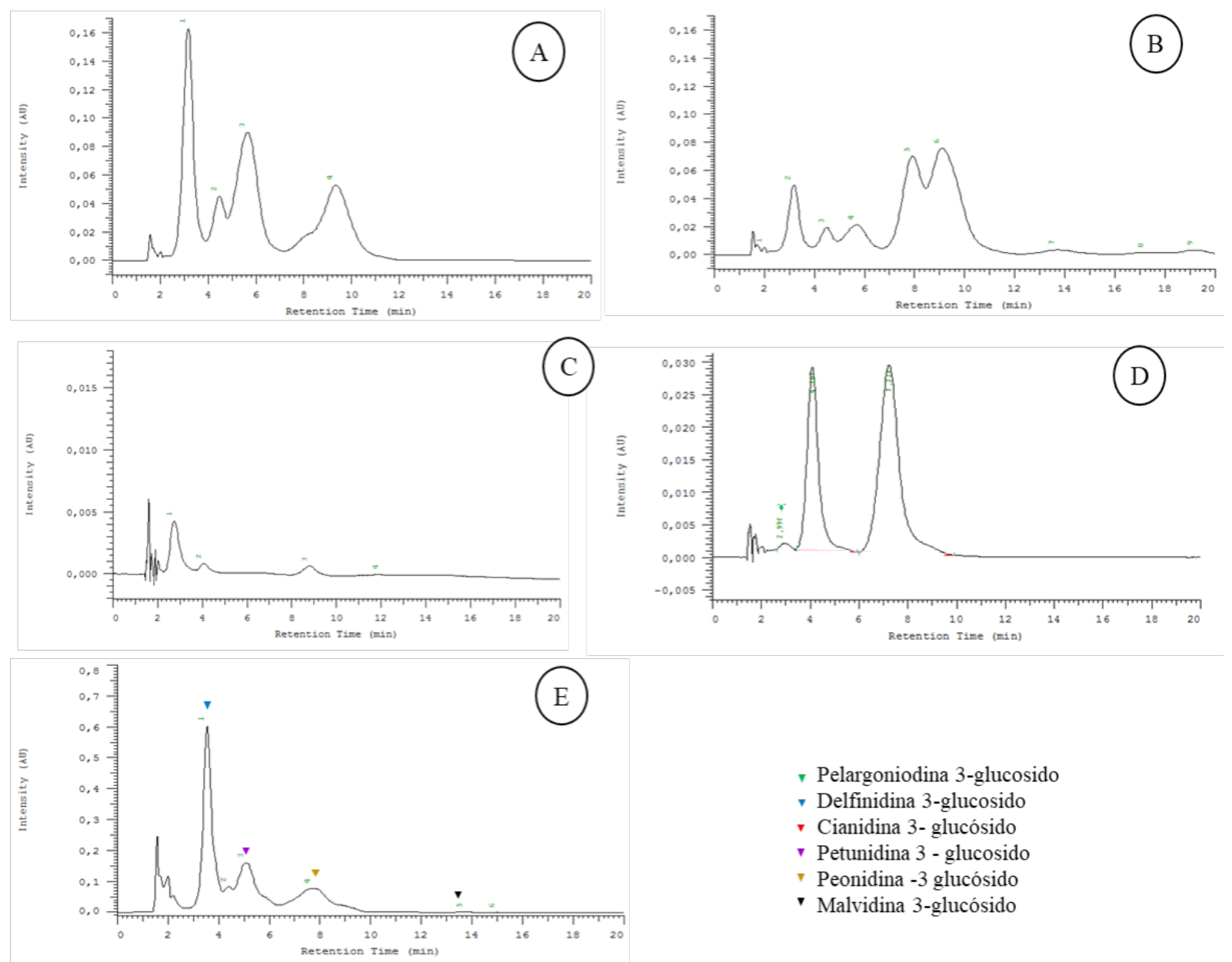

Figura 3. Cromatogramas HPLC-DAD, longitud de onda $520 \mathrm{~nm}$. A Arrayan (L. apiculata) B. Tintorera (V. vinifera) C. Frutilla blanca (F. chiloensis ssp. chiloensis) D. murtilla (U. molinae) y E. calafate (B. microphylla).

En el presente estudio para los 5 berries mediante HPLC-DAD se identificaron 9 antocianinas a partir de extractos, todas ellas variantes glucosiladas de cianidina, delfinidina, malvidina, peonidina, petunidina y pelargonidina. Los cromatogramas individuales constan en la Figura 3. Estos resultados son similares a los reportados en otras investigaciones donde se menciona que los compuestos predominantes en todos los berries comestibles son malvidina-3-glucósido, cumarato de malvidina-3-glucósido y la delfinidina-3-glucósido (24) (17) y se destaca este último presente en calafate (Fig. 3-E) que se encuentra en mayor cantidad respecto a las otras bayas (27).

\section{DISCUSIÓN}

Según la FAO, en frutas el pH 3,5 es el mínimo requerido para la aceptación y consumo (29); en consecuencia, todas las frutas analizadas en este trabajo pueden tener alta probabilidad de ser aceptadas por los consumidores. Los grados Brix (entre 5 y 20) y alto contenido de azúcares totales son indicadores de madurez y calidad gustativa (30) en este calafate y uva tintorera sobresalen. Por otro lado, algunas antocianinas se enlazan con diferentes azúcares (2), lo que indica que la presencia de altos contenidos de azúcar podría potenciar el efecto de dichos flavonoides frente al estrés oxidativo.

La caracterización de diferentes tipos de frutas y su contenido de componentes antioxidantes específicos responde a la orientación actual de consumo de alimentos que contengan compuestos bioactivos y presenten beneficios a la salud (3). Los resultados respecto a polifenoles y antocianinas en calafate mostraron valores superiores a otros reportes (24). Así mismo, se pudo constatar que todos los berries en estudio poseen contenidos de compuestos fenólicos considerables, incluso superiores a los resultados reportados para bayas comerciales como en el caso de uva tintorera $(639,5 \pm 41,8)$ frente a lo probado para uvas comerciales 460,45 $\pm 45 \mathrm{mg}$ ácido gálico /100g de muestra (17). 
Así, el poder reductor de estas bayas es promisorio. Las diferencias en las cuantificaciones analíticas, respecto al poder reductor de las frutas nativas chilenas comparado con los valores obtenidos por otros investigadores se debe a que la cantidad de polifenoles totales como de antocianinas totales depende de diversos factores medio ambientales, localidad de recolección, etapa de maduración, especie, tipo de cultivo, entre otros (31). De igual forma, trabajos previos $(9,10,32)$ demuestran que las antocianinas tienen efectos beneficiosos para el tratamiento de enfermedades crónicas, por ello, la referencia en término de estos compuestos es importante para continuar con estudios tanto in vitro como in vivo respecto a las propiedades neuroprotectoras, cardioprotectoras, antiinflamatorias y anticancerígenas que estos berries pudieran ofrecer.

\section{CONCLUSIONES}

Estos resultados preliminares indican que los perfiles de antocianinas de las cinco 5 bayas recolectadas de la región centro y sur de Chile mostraron un alto predominio de antocianinas (delfinidina y derivados de cianidina). Calafate (B. microphylla) es la baya que mostró los niveles más altos de antocianinas totales y actividad antioxidante por el método FRAP y DPPH seguida de uva tintorera y arrayan. Por su parte murtilla y frutilla blanca, a pesar de mostrar cantidades menores de antocianinas y polifenoles, gracias al estudio del perfil químico, se encontraron compuestos que podrían ser de interés para estudios posteriores como los elagitaninos en frutilla blanca (F. chiloensis ssp. chiloensis). Estos frutos ricos en compuestos polifenólicos tienen un futuro prometedor para ser incorporados como ingredientes funcionales, por lo que es meritorio continuar con el estudio de estas bayas.

\section{AGRADECIMIENTOS}

Reconocimiento y gratitud al Laboratorio de Análisis Químico del Departamento de Producción Vegetal de la Universidad de Concepción, campus Chillán, a CONICYT FONDECYT 1160899 de Chile y, al Instituto de Fomento al Talento Humano y Secretaria de Educación Superior de Ciencia, Tecnología e Innovación de Ecuador.

\section{$\mathrm{R}$}

1. Blasa M, Gennari L, Angelino D, Ninfali P. Chapter 3 - Fruit and Vegetable Antioxidants in Health. In: Watson RR, Preedy VR, editors. Bioactive Foods in Promoting Health [Internet]. San Diego: Academic Press; 2010. p. 37-58. Available from: http://www.sciencedirect.com/science/article/pii/B9780123746283000037

2. Amalesh S, Kumar S, Das G. Roles of flavonoids in Plants. ResearchGate [Internet]. 2011 [cited 2018 Apr 23]; Available from: https://www.researchgate.net/publication/279499208_Roles_of_flavonoids_in_Plants

3. Panche AN, Diwan AD, Chandra SR. Flavonoids: an overview. J Nutr Sci [Internet]. 2016 Dec 29 [cited 2018 Apr 24];5. Available from: https:/www.ncbi.nlm.nih.gov/pmc/articles/PMC5465813/

4. Ncube B, Van Staden J. Tilting Plant Metabolism for Improved Metabolite Biosynthesis and Enhanced Human Benefit. Molecules. 2015 Jul 13;20(7):12698-731.

5. Mewis I, Schreiner M, Nguyen CN, Krumbein A, Ulrichs C, Lohse M, et al. UV-B Irradiation Changes Specifically the Secondary Metabolite Profile in Broccoli Sprouts: Induced Signaling Overlaps with Defense Response to Biotic Stressors. Plant Cell Physiol. 2012 Sep;53(9):1546-60.

6. Eichholz I, Huyskens-Keil S, Keller A, Ulrich D, Kroh LW, Rohn S. UV-B-induced changes of volatile metabolites and phenolic compounds in blueberries (Vaccinium corymbosum L.). Food Chem [Internet]. 2011; Available from: http://agris.fao.org/agris-search/search.do?recordID=US201301920818

7. Neal M, Richardson JR. Time to get personal: A framework for personalized targeting of oxidative stress in neurotoxicity and neurodegenerative disease. Curr Opin Toxicol. 2018 Feb 1;7:127-32.

8. Walker LC, Jucker M. Neurodegenerative Diseases: Expanding the Prion Concept. Annu Rev Neurosci. 2015;38(1):87-103.

9. Yang L, Ling W, Du Z, Chen Y, Li D, Deng S, et al. Effects of Anthocyanins on Cardiometabolic Health: A Systematic Review and Meta-Analysis of Randomized Controlled Trials. Adv Nutr Bethesda Md. 2017 Sep;8(5):684-93.

10. Spagnuolo C, Moccia S, Russo GL. Anti-inflammatory effects of flavonoids in neurodegenerative disorders. Eur J Med Chem. 2018 Jun 10;153:105-15.

11. Varas B, Castro MH, Rodriguez R, von Baer D, Mardones C, Hinrichsen P. Identification and characterization of microsatellites from calafate (Berberis microphylla, Berberidaceae)1. Appl Plant Sci [Internet]. 2013 
Jul 5 [cited 2018 May 5];1(7). Available from: https://www.ncbi.nlm.nih.gov/pmc/articles/PMC4103124/

12. Simirgiotis MJ, Schmeda-Hirschmann G. Determination of phenolic composition and antioxidant activity in fruits, rhizomes and leaves of the white strawberry (Fragaria chiloensis spp. chiloensis form chiloensis) using HPLC-DAD-ESI-MS and free radical quenching techniques. J Food Compos Anal. 2010;23(6):545-53. 13. Mariangel E, Reyes-Diaz M, Lobos W, Bensch E, Schalchli H, Ibarra P. The antioxidant properties of calafate (Berberis microphylla) fruits from four different locations in southern Chile. Cienc E Investig Agrar. 2013 Apr;40(1):161-70.

14. Simirgiotis MJ, Bórquez J, Schmeda-Hirschmann G. Antioxidant capacity, polyphenolic content and tandem HPLC-DAD-ESI/MS profiling of phenolic compounds from the South American berries Luma apiculata and L. chequén. Food Chem. 2013 Aug 15;139(1):289-99.

15. Junqueira-Gonçalves MP, Yáñez L, Morales C, Navarro M, A. Contreras R, Zúñiga GE. Isolation and Characterization of Phenolic Compounds and Anthocyanins from Murta (Ugni molinae Turcz.) Fruits. Assessment of Antioxidant and Antibacterial Activity. Molecules. 2015 Mar 31;20(4):5698-713.

16. Figueiredo-González M, Regueiro J, Cancho-Grande B, Simal-Gándara J. Garnacha Tintorera-based sweet wines: Detailed phenolic composition by HPLC/DAD-ESI/MS analysis. Food Chem. 2014 Jan 15;143:282-92. 17. Burin B, Falcao L, Valdemoro L. Color, contenido fenólico y actividad antioxidante del jugo de uva. Ciênc Tecnol Aliment. 2010;30(4):1-6.

18. Lee J, Durst RW, Wrolstad RE. Determination of total monomeric anthocyanin pigment content of fruit juices, beverages, natural colorants, and wines by the $\mathrm{pH}$ differential method: collaborative study. J AOAC Int. 2005 Oct;88(5):1269-78.

19. Prior RL, Lazarus SA, Cao G, Muccitelli H, Hammerstone JF. Identification of Procyanidins and Anthocyanins in Blueberries and Cranberries (Vaccinium Spp.) Using High-Performance Liquid Chromatography/ Mass Spectrometry. J Agric Food Chem. 2001 Mar 1;49(3):1270-6.

20. Aaby K, Skrede G, Wrolstad R. Antioxidant Activities in Flesh and Achenes of Strawberries (Fragaria ananassa). J Agric Food Chem. 2005;53(10):4032-40.

21. Mathew M, Subramanian S. In Vitro Screening for Anti-Cholinesterase and Antioxidant Activity of Methanolic Extracts of Ayurvedic Medicinal Plants Used for Cognitive Disorders. PLOS ONE. 2014 Jan 23;9(1):e86804.

22. Singleton VL, Rossi JA. Colorimetry of Total Phenolics with Phosphomolybdic-Phosphotungstic Acid Reagents. Am J Enol Vitic. 1965 Jan 1;16(3):144-58.

23. Fuleki T, Francis F. Quantitative methods for anthocyanins. 1. Extraction and determination of total anthocyanin in cranberries. J Food Sci. 1968;33(1):72-7.

24. Brito A, Areche C, Sepúlveda B, Kennelly EJ, Simirgiotis MJ. Anthocyanin Characterization, Total Phenolic Quantification and Antioxidant Features of Some Chilean Edible Berry Extracts. Molecules. 2014 Jul 28;19(8):10936-55.

25. Castro-López C, Rojas R, Sánchez-Alejo EJ, Niño-Medina G, Martínez-Ávila GCG. Phenolic Compounds Recovery from Grape Fruit and By- Products: An Overview of Extraction Methods. In: Morata A, Loira I, editors. Grape and Wine Biotechnology [Internet]. Rijeka: InTech; 2016 [cited 2018 Mar 13]. p. Ch. 05. Available from: http://dx.doi.org/10.5772/64821

26. Ruiz A, Bustamante L, Vergara C, von Baer D, Hermosín-Gutiérrez I, Obando L, et al. Hydroxycinnamic acids and flavonols in native edible berries of South Patagonia. Food Chem. 2015 Jan 15;167:84-90.

27. Lopez MD, Romero ME, Vera B. El calafate. Su industrialización abre nuevas perspectivas. Indualimentos [Internet]. 2018 abil;110. Available from: http://www.indualimentos.cl/edic.html

28. Larrosa M, García-Conesa MT, Espín JC, Tomás-Barberán FA. Ellagitannins, ellagic acid and vascular health. Mol Aspects Med. 2010 Dec 1;31(6):513-39.

29. FAO. Agricultura mundial: hacia los años 2015/2030 [Internet]. 2015 [cited 2017 Jul 27]. Available from: http://www.fao.org/docrep/004/y3557s/y3557s06.htm

30. Cárdenas G, Arrazola G, Villalba M. Frutas tropicales: fuente de compuestos bioactivos naturales en la industria de alimentos-Tropical Fruits: Source of Natural Compounds Bioactives Food Industry | Cárdenas Baquero | Ingenium Revista de la facultad de ingeniería. 2016 [cited 2017 Nov 28]; Available from: http://revistas.usb.edu.co/index.php/Ingenium/article/view/2152

31. Ruiz A, Hermosín-Gutiérrez I, Vergara C, von Baer D, Zapata M, Hitschfeld A, et al. Anthocyanin profiles in south Patagonian wild berries by HPLC-DAD-ESI-MS/MS. Food Res Int. 2013 May 1;51(2):706-13.

32. Wightman JD, Heuberger RA. Effect of grape and other berries on cardiovascular health. J Sci Food Agric. 2015;95(8):1584-97. 